\title{
Oscillation criteria for second-order nonlinear dynamic equations
}

\section{Taher S Hassan*}

\section{*Correspondence:}

tshassan@mans.edu.eg

Department of Mathematics,

Faculty of Science, Mansoura

University, Mansoura, 35516, Egypt

\begin{abstract}
This paper concerns the oscillation of solutions to the second-order dynamic equation

$$
\left(r(t) x^{\Delta}(t)\right)^{\Delta}+p(t) x^{\Delta}(t)+q(t) f\left(x^{\sigma}(t)\right)=0,
$$

on a time scale $\mathbb{T}$ which is unbounded above. No sign conditions are imposed on $r(t)$, $p(t)$, and $q(t)$. The function $f \in C(\mathbb{R}, \mathbb{R})$ is assumed to satisfy $x f(x)>0$ and $f^{\prime}(x)>0$ for $x \neq 0$. In addition, there is no need to assume certain restrictive conditions and also the both cases

$$
\int_{t_{0}}^{\infty} \frac{\Delta t}{r(t)}=\infty \text { and } \quad \int_{t_{0}}^{\infty} \frac{\Delta t}{r(t)}<\infty
$$
\end{abstract}

are considered. Our results will improve and extend results in (Baoguo et al. in Can. Math. Bull. 54:580-592, 2011; Bohner et al. in J. Math. Anal. Appl. 301:491-507, 2005; Hassan et al. in Comput. Math. Anal. 59:550-558, 2010; Hassan et al. in J. Differ. Equ. Appl. 17:505-523, 2011) and many known results on nonlinear oscillation. These results have significant importance to the study of oscillation criteria on discrete time scales such as $\mathbb{T}=\mathbb{Z}, \mathbb{T}=h \mathbb{Z}, h>0$, or $\mathbb{T}=\left\{t: t=q^{k}, k \in \mathbb{N}_{0}, q>1\right\}$ and the space of harmonic numbers $\mathbb{T}=H_{n}$. Some examples illustrating the importance of our results are also included.

MSC: 34K11; 39A10; 39A99

Keywords: oscillation; second order; dynamic equations; time scales

\section{Introduction}

The theory of time scales, which has recently received a lot of attention, was introduced by Stefan Hilger in his $\mathrm{PhD}$ dissertation written under the direction of Bernd Aulbach (see [1]). Since then a rapidly expanding body of literature has sought to unify, extend, and generalize ideas from discrete calculus, quantum calculus, and continuous calculus to arbitrary time scale calculus. Recall that a time scale $\mathbb{T}$ is a nonempty closed subset of the reals, and the cases when this time scale is the reals or the integers represent the classical theories of differential and of difference equations. Not only does the new theory of the so-called 'dynamic equations' unify the theories of differential equations and difference equations, but it also extends these classical cases to cases 'in between', e.g., to the socalled $q$-difference equations when $\mathbb{T}=q^{\mathbb{N} 0}$, and can be applied to different types of time

() 2012 Hassan; licensee Springer. This is an Open Access article distributed under the terms of the Creative Commons Attribution License (http://creativecommons.org/licenses/by/2.0), which permits unrestricted use, distribution, and reproduction in any medium, provided the original work is properly cited. 
scales like $\mathbb{T}=h \mathbb{Z}, \mathbb{T}=\mathbb{N}_{0}^{2}$, and the space of harmonic numbers $\mathbb{T}=\left\{\mathbb{H}_{n}\right\}$. In this work, knowledge and understanding of time scales and time scale notation is assumed. For an excellent introduction to the calculus on time scales, see Bohner and Peterson [2,3]. We recall the following concepts related to the notion of time scales. A time scale $\mathbb{T}$ is an arbitrary nonempty closed subset of the real numbers $\mathbb{R}$. For $t \in \mathbb{T}$, we define the forward and backward jump operators $\sigma: \mathbb{T} \rightarrow \mathbb{T}$ and $\rho: \mathbb{T} \rightarrow \mathbb{T}$ by

$$
\sigma(t)=\inf \{s \in \mathbb{T}: s>t\}, \quad \rho(t)=\sup \{s \in \mathbb{T}: s<t\},
$$

where $\inf \emptyset:=\sup \mathbb{T}$ and $\sup \emptyset=\inf \mathbb{T}$, where $\emptyset$ denotes the empty set. A point $t \in \mathbb{T}, t>$ $\inf \mathbb{T}$, is said to be left-dense if $\rho(t)=t$, right-dense if $t<\sup \mathbb{T}$ and $\sigma(t)=t$, left-scattered if $\rho(t)<t$, and right-scattered if $\sigma(t)>t$. A function $g: \mathbb{T} \rightarrow \mathbb{R}$ is said to be right-dense continuous (rd-continuous) provided $g$ is continuous at right-dense points and at leftdense points in $\mathbb{T}$, left-hand limits exist and are finite. The set of all such rd-continuous functions is denoted by $C_{\mathrm{rd}}(\mathbb{T})$.

We are concerned with the oscillatory behavior of the following second-order dynamic equation

$$
\left(r(t) x^{\Delta}(t)\right)^{\Delta}+p(t) x^{\Delta}(t)+q(t) f\left(x^{\sigma}(t)\right)=0,
$$

on a time scale $\mathbb{T}$ which is unbounded above, where $r, p$, and $q$ are real-valued, right-dense continuous functions on $\mathbb{T}$. The function $f \in C(\mathbb{R}, \mathbb{R})$ is assumed to satisfy $x f(x)>0$ and $f^{\prime}(x)>0$ for $x \neq 0$.

By a solution of (1.1), we mean a nontrivial real-valued function $x \in C_{\mathrm{rd}}^{1}\left[T_{x}, \infty\right), T_{x} \geq t_{0}$ which has the property that $r x^{\Delta} \in C_{\mathrm{rd}}^{1}\left[T_{x}, \infty\right)$ and satisfies equation (1.1) on $\left[T_{x}, \infty\right)$, where $C_{\text {rd }}$ is the space of rd-continuous functions. The solutions vanishing in some neighborhood of infinity will be excluded from our consideration. A solution $x$ of (1.1) is said to be oscillatory if it is neither eventually positive nor eventually negative. Otherwise it is said to be nonoscillatory. There has been a great deal of research into obtaining criteria for oscillation of all solutions of dynamic equations on time scales. It is usually assumed that $r, p, q$ are nonnegative functions. We refer the reader to the papers [4-11, 22-33] and the references cited therein. On the other hand, very little is known about equations when no explicit sign assumptions are made with respect to the coefficient functions $p, q$, and $r$. In the papers [12-14], it is shown that one may relate oscillation and boundedness of solutions of the nonlinear equation (1.1) to a related linear equation, which, in the case $r=1$ and $p=0$, reduces to

$$
x^{\Delta \Delta}(t)+\lambda q(t) x^{\sigma}(t)=0,
$$

where $\lambda>0$, for which many oscillation criteria are known. However, it was assumed that the nonlinearity has the property

$$
f^{\prime}(x) \geq \frac{f(x)}{x}, \quad \text { for } x \neq 0 .
$$

Bohner, Erbe, and Peterson [15] studied the second-order nonlinear dynamic equation

$$
x^{\Delta \Delta}(t)+p(t) x^{\Delta \sigma}(t)+q(t) f\left(x^{\sigma}(t)\right)=0
$$


where $p$ is a positively regressive function and $q_{1}$ satisfies condition (A), that is,

$$
\liminf _{t \rightarrow \infty} \int_{T}^{t} q_{1}(s) \Delta s \geq 0 \quad \text { and } \quad \not \equiv 0
$$

for all large $T$ and

$$
\int_{t_{0}}^{\infty} \frac{\Delta t}{r_{1}(t)}=\int_{t_{0}}^{\infty} q_{1}(t) \Delta t=\infty
$$

where $q_{1}(t):=q(t) e_{p}\left(t, t_{0}\right)$ and $r_{1}(t):=e_{p}\left(t, t_{0}\right)$. Oscillation criterion for equation (1.2) is shown in [15] when $f^{\prime}$ satisfied condition (B), if for each $k>0$ there exists $m>0$ such that $f^{\prime}(x) \geq m$ provided $x \geq k$. We say $\mathbb{T}$ satisfies condition (C) if there is an $M>0$ such that $\chi(t) \leq M \mu(t), t \in \mathbb{T}$, where $\chi$ is the characteristic function of the set $\hat{\mathbb{T}}=\{t \in \mathbb{T}: \mu(t)>0\}$. We note that if $\mathbb{T}$ satisfies condition (C), then the subset $\check{\mathbb{T}}$ of $\mathbb{T}$ defined by

$$
\check{\mathbb{T}}=\{t \in \mathbb{T}: t>0 \text { is right-scattered or left-scattered }\}
$$

is necessarily countable and $\hat{\mathbb{T}} \subset \check{\mathbb{T}}$. Then we can rewrite $\check{\mathbb{T}}$ by

$$
\check{\mathbb{T}}=\left\{t_{i} \in \mathbb{T}: 0<t_{1}<t_{2}<\cdots<t_{n}<\cdots\right\},
$$

and so

$$
\mathbb{T}=\check{\mathbb{T}} \cup\left[\bigcup_{n \in A}\left(t_{n-1}, t_{n}\right)\right]
$$

where $A$ is the set of all integers for which the real open interval $\left(t_{n-1}, t_{n}\right)$ is contained in $\mathbb{T}$. To be precise, we have

$$
A:=\left\{n \in \mathbb{N}:\left(t_{n-1}, t_{n}\right) \subset \mathbb{T}\right\}
$$

Baoguo, Erbe, and Peterson established in [16] some oscillation criteria of Kiguradze-type in particular, for the second-order superlinear dynamic equation

$$
x^{\Delta \Delta}(t)+q(t) f\left(x^{\sigma}(t)\right)=0,
$$

where $\mathbb{T}$ satisfies condition $(\mathrm{C})$ and $f(x)$ satisfies the superlinearity condition

$$
0<\int_{\epsilon}^{\infty} \frac{d x}{f(x)}, \quad \int_{-\infty}^{-\epsilon} \frac{d x}{f(x)}<\infty, \quad \text { for all } \epsilon>0 .
$$

Hassan, Erbe, and Peterson [17] improved these results and generalized these to the superlinear dynamic equation (1.1), where the coefficient functions $r, p, q$ are allowed to change sign for large $t$, and $\mathbb{T}$ satisfies condition (C). Also, Hassan, Erbe, and Peterson [18] applied these results to the sublinear dynamic equation (1.1), where the functions $r, p, q$ are 
also allowed to change sign for large $t$, and $\mathbb{T}$ satisfies condition $(C)$, and $f(x)$ satisfies the sublinearity condition

$$
0<\int_{0}^{\epsilon} \frac{d x}{f(x)} ; \quad \int_{-\epsilon}^{0} \frac{d x}{f(x)}<\infty, \quad \text { for all } \epsilon>0 .
$$

A number of sufficient conditions for oscillation were obtained in $[15,16]$ for the case when

$$
\int_{t_{0}}^{\infty} \frac{\Delta t}{r(t)}=\infty
$$

and in $[17,18]$ for the case when

$$
\int_{t_{0}}^{\infty} \frac{\Delta t}{\phi(t) r(t)}=\infty
$$

where $\phi$ is a rd-continuous function. Therefore, it will be of great interest to establish oscillation criteria for (1.1) for both of the cases

$$
\int_{t_{0}}^{\infty} \frac{\Delta t}{\phi(t) r(t)}=\infty
$$

and

$$
\int_{t_{0}}^{\infty} \frac{\Delta t}{\phi(t) r(t)}<\infty
$$

where the function $\phi$ will be defined in the next section. We will still assume that the functions $r(t), p(t)$, and $q(t)$ change sign for arbitrarily large values of $t$, and conditions (A), (B), and (C) are not needed. Hence, our results will improve and extend results in [15-18] and many known results on nonlinear oscillation. In addition, linear, sublinear, and superlinear results will be presented.

These results have significant importance to the study of oscillation criteria on discrete time scales such as $\mathbb{T}=\mathbb{Z}, \mathbb{T}=h \mathbb{Z}, h>0$, or $\mathbb{T}=\left\{t: t=q^{k}, k \in \mathbb{N}_{0}, q>1\right\}$ and the space of harmonic numbers $\mathbb{T}=H_{n}$. In particular, we give examples where the coefficient function $r$ changes sign for large $t$ as well as $p$ and $q$ and without conditions (1.3) and (1.4).

\section{Main results}

Before stating our main results, we begin with the following lemma (Second Mean Value Theorem) which will play an important role in the proof of our main results.

Lemma 2.1 ([3, Theorem 5.45]) Let $h$ be a bounded function that is integrable on $[a, b]_{\mathbb{T}}$. Let $m_{H}$ and $M_{H}$ be the infimum and supremum of the function $H(t):=\int_{a}^{t} h(s) \Delta s$ on $[a, b]_{\mathbb{T}}$ respectively. Suppose that $g$ is a nonnegative and nonincreasing function on $[a, b]_{\mathbb{T}}$. Then there is some number $\Lambda$ with $m_{H} \leq \Lambda \leq M_{H}$ such that

$$
\int_{a}^{b} h(t) g(t) \Delta t=g(a) \Lambda
$$

Our first result is stated in terms of an auxiliary function $\phi=\phi(t)$. 
Theorem 2.1 Assume that $f$ satisfies (1.3). If there exists a $C_{\mathrm{rd}}^{1}$ function $\phi$ such that

$$
\phi(t) r(t)>0, \quad P(t) \geq 0, \quad P^{\Delta}(t) \leq 0, \quad \text { for } t \in\left[t_{0}, \infty\right)_{\mathbb{T}},
$$

where $P(t):=\phi^{\Delta}(t) r(t)-\phi^{\sigma}(t) p(t)$ and

$$
\int_{t_{0}}^{\infty} \frac{\Delta t}{\phi(t) r(t)}=\int_{t_{0}}^{\infty} \phi^{\sigma}(t) q(t) \Delta t=\infty
$$

then every solution of equation (1.1) is oscillatory.

Proof Assume (1.1) has a nonoscillatory solution $x$ on $\left[t_{0}, \infty\right)_{\mathbb{T}}$. Then, without loss of generality, there is a solution $x$ of $(1.1)$ and a $T \in\left[t_{0}, \infty\right)_{\mathbb{T}}$ such that $x(t)>0$ on $[T, \infty)_{\mathbb{T}}$. Define

$$
w(t):=\frac{\phi(t) r(t) x^{\Delta}(t)}{f(x(t))} .
$$

Then from the product and quotient rules and the Pötzsche chain rule [2, Theorem 1.90], we get

$$
\begin{aligned}
w^{\Delta}(t)= & {\left[\frac{\phi(t)}{f(x(t))}\right]^{\sigma}\left(r(t) x^{\Delta}(t)\right)^{\Delta}+\left[\frac{\phi(t)}{f(x(t))}\right]^{\Delta} r(t) x^{\Delta}(t) } \\
= & {\left[\frac{\phi(t)}{f(x(t))}\right]^{\sigma}\left(r(t) x^{\Delta}(t)\right)^{\Delta} } \\
& +\left[\frac{\phi^{\Delta}(t)}{f\left(x^{\sigma}(t)\right)}-\frac{\phi(t) \int_{0}^{1} f^{\prime}\left(x_{h}(t)\right) d h x^{\Delta}(t)}{f(x(t)) f\left(x^{\sigma}(t)\right)}\right] r(t) x^{\Delta}(t),
\end{aligned}
$$

where $x_{h}(t):=(1-h) x(t)+h x^{\sigma}(t)>0$ for $0 \leq h \leq 1, t \in[T, \infty)_{\mathbb{T}}$. Therefore, from (1.1), we have

$$
w^{\Delta}(t)=-\phi^{\sigma}(t) q(t)+\frac{P(t) x^{\Delta}(t)}{f\left(x^{\sigma}(t)\right)}-\frac{\phi(t) r(t) \int_{0}^{1} f^{\prime}\left(x_{h}(t)\right) d h\left(x^{\Delta}(t)\right)^{2}}{f(x(t)) f\left(x^{\sigma}(t)\right)} .
$$

Since $v f(v)>0, f^{\prime}(v)>0$, for all $v \neq 0$ and $\phi(t) r(t)>0$, for all $t \geq T$, we get

$$
w^{\Delta}(t) \leq-\phi^{\sigma}(t) q(t)+\frac{P(t) x^{\Delta}(t)}{f\left(x^{\sigma}(t)\right)}, \quad \text { for } t \in[T, \infty)_{\mathbb{T}} .
$$

Integrating the above inequality from $T$ to $t(\geq T)$, we obtain

$$
w(t) \leq w(T)-\int_{T}^{t} \phi^{\sigma}(s) q(s) \Delta s+\int_{T}^{t} \frac{P(s) x^{\Delta}(s)}{f\left(x^{\sigma}(s)\right)} \Delta s
$$

We claim that $\int_{T}^{t} \frac{P(s) x^{\Delta}(s)}{f\left(x^{\sigma}(s)\right)} \Delta s$ is bounded above for all $t \geq T$. Since $P(t) \geq 0$ and $P^{\Delta}(t) \leq 0$, we have from Lemma 2.1 that for each $t \in[T, \infty)_{\mathbb{T}}$,

$$
\int_{T}^{t} \frac{P(s) x^{\Delta}(s)}{f\left(x^{\sigma}(s)\right)} \Delta s=P(T) \Lambda(t)
$$


where $m \leq \Lambda(t) \leq M$, and where $m$ and $M$ denote the infimum and supremum, respectively, of the function $\int_{T}^{s} \frac{x^{\Delta}(\tau)}{f\left(x^{\sigma}(\tau)\right)} \Delta \tau$ for $s \in[T, t)_{\mathbb{T}}$. Define

$$
F(x(s)):=\int_{x(T)}^{x(s)} \frac{d \tau}{f(\tau)}
$$

and so

$$
(F(x(s)))^{\Delta}=\int_{0}^{1} F^{\prime}\left(x_{h}(s)\right) d h x^{\Delta}(s)=\int_{0}^{1} \frac{1}{f\left(x_{h}(s)\right)} d h x^{\Delta}(s) .
$$

For a fixed point $s \in[T, \infty)_{\mathbb{T}}$, we have

$$
x_{h}(s)=(1-h) x(s)+h x^{\sigma}(s) \begin{cases}\geq x^{\sigma}(s), & \text { if } x^{\Delta}(s) \leq 0, \\ \leq x^{\sigma}(s), & \text { if } x^{\Delta}(s) \geq 0,\end{cases}
$$

and so

$$
\frac{x^{\Delta}(s)}{f\left(x_{h}(s)\right)} \geq \begin{cases}\frac{x^{\Delta}(s)}{f\left(x^{\sigma}(s)\right)}, & \text { if } x^{\Delta}(s) \leq 0, \\ \frac{x^{\Delta}(s)}{f\left(x^{\sigma}(s)\right)}, & \text { if } x^{\Delta}(s) \geq 0 .\end{cases}
$$

Then

$$
\frac{x^{\Delta}(s)}{f\left(x_{h}(s)\right)} \geq \frac{x^{\Delta}(s)}{f\left(x^{\sigma}(s)\right)}, \quad \text { for } s \in[T, \infty)_{\mathbb{T}} .
$$

And so, from (2.6), we have

$$
(F(x(s)))^{\Delta} \geq \frac{x^{\Delta}(s)}{f\left(x^{\sigma}(s)\right)}, \quad \text { for } s \in[T, \infty)_{\mathbb{T}} .
$$

Also,

$$
F(x(s))=\int_{x(T)}^{x(s)} \frac{d \tau}{f(\tau)}< \begin{cases}0, & \text { if } x(s) \leq x(T), \\ \int_{x(T)}^{\infty} \frac{d \tau}{f(\tau)}, & \text { if } x(s)>x(T) .\end{cases}
$$

Then, from (1.3), we get

$$
F(x(s))=\int_{x(T)}^{x(s)} \frac{d \tau}{f(\tau)} \leq L_{1}, \quad \text { for } s \in[T, \infty)_{\mathbb{T}} .
$$

Hence, it follows that

$$
\int_{T}^{t} \frac{x^{\Delta}(s)}{f\left(x^{\sigma}(s)\right)} \Delta s \leq F(x(t))-F(x(T)) \leq L_{1},
$$

and from (2.5), for all $t \in[T, \infty)_{\mathbb{T}}$, we have

$$
\int_{T}^{t} \frac{P(s) x^{\Delta}(s)}{f\left(x^{\sigma}(s)\right)} \Delta s=P(T) \Lambda(t) \leq P(T) L_{1} .
$$


From (2.4) and (2.7), we get for $t \in[T, \infty)_{\mathbb{T}}$,

$$
w^{\Delta}(t) \leq w^{\Delta}(T)+P(T) L_{1}-\int_{T}^{t} \phi^{\sigma}(s) q(s) \Delta s
$$

In view of condition (2.2), it follows from the last inequality that there exists a sufficiently large $T_{1} \geq T$ such that

$$
x^{\Delta}(t)<0, \quad \text { for } t \in\left[T_{1}, \infty\right)_{\mathbb{T}} .
$$

Also, from (2.2), there exists $T_{2} \geq T_{1}$ such that

$$
\int_{T_{2}}^{t} \phi^{\sigma}(s) q(s) \Delta s \geq 0, \quad \text { for all } t \geq T_{2} .
$$

Indeed, (1.1) yields on integration

$$
\begin{gathered}
\int_{T_{2}}^{t} \phi^{\sigma}(s)\left(r(s) x^{\Delta}(s)\right)^{\Delta} \Delta s+\int_{T_{2}}^{t} \phi^{\sigma}(s) p(s) x^{\Delta}(s) \Delta s \\
+\int_{T_{2}}^{t} \phi^{\sigma}(s) q(s) f\left(x^{\sigma}(s)\right) \Delta s=0 .
\end{gathered}
$$

Now by the integration by parts, we have

$$
\begin{gathered}
\int_{T_{2}}^{t} \phi^{\sigma}(s)\left(r(s) x^{\Delta}(s)\right)^{\Delta} \Delta s+\int_{T_{2}}^{t} \phi^{\sigma}(s) p(s) x^{\Delta}(s) \Delta s \\
=\phi(t) r(t) x^{\Delta}(t)-\phi\left(T_{2}\right) r\left(T_{2}\right) x^{\Delta}\left(T_{2}\right) \\
\quad-\int_{T_{2}}^{t} \phi^{\Delta}(s) r(s) x^{\Delta}(s) \Delta s+\int_{T_{2}}^{t} \phi^{\sigma}(s) p(s) x^{\Delta}(s) \Delta s \\
=\phi(t) r(t) x^{\Delta}(t)-\phi\left(T_{2}\right) r\left(T_{2}\right) x^{\Delta}\left(T_{2}\right)-\int_{T_{2}}^{t} P(s) x^{\Delta}(s) \Delta s \\
\geq \phi(t) r(t) x^{\Delta}(t)-\phi\left(T_{2}\right) r\left(T_{2}\right) x^{\Delta}\left(T_{2}\right),
\end{gathered}
$$

and by the integration by parts and the Pötzsche chain rule and then by (2.8), we get

$$
\begin{aligned}
\int_{T_{2}}^{t} \phi^{\sigma}(s) q(s) f\left(x^{\sigma}(s)\right) \Delta s= & f(x(t)) \int_{T_{2}}^{t} \phi^{\sigma}(\tau) q(\tau) \Delta \tau \\
& -\int_{T_{2}}^{t}\left[\int_{0}^{1} f^{\prime}\left(x_{h}(s)\right) d h\right] x^{\Delta}(s) \int_{T_{2}}^{s} \phi^{\sigma}(\tau) q(\tau) \Delta \tau \Delta s \\
\geq & f(x(t)) \int_{T_{2}}^{t} \phi^{\sigma}(s) q(s) \Delta s \geq 0, \quad \text { for } t \in\left[T_{2}, \infty\right)_{\mathbb{T}},
\end{aligned}
$$

since $v f(v)>0$ and $f^{\prime}(v)>0$ for all $v \neq 0$ and $x^{\Delta}(t)<0$ for $t \geq T_{2}$. Using (2.9) and (2.10) in (2.11), we get

$$
\phi(t) r(t) x^{\Delta}(t) \leq \phi\left(T_{2}\right) r\left(T_{2}\right) x^{\Delta}\left(T_{2}\right),
$$


and so

$$
x(t)-x\left(T_{2}\right) \leq \phi\left(T_{2}\right) r\left(T_{2}\right) x^{\Delta}\left(T_{2}\right) \int_{T_{2}}^{t} \frac{\Delta s}{\phi(s) r(s)} .
$$

Since $\phi\left(T_{2}\right) r\left(T_{2}\right) x^{\Delta}\left(T_{2}\right)<0$, we conclude from (2.2) that $\lim _{t \rightarrow \infty} x(t)=-\infty$, which is a contradiction. This completes the proof.

In the case $\mathbb{T}=\mathbb{R}, r(t)=1, p(t)=0$, and $f(x)=x^{\alpha}, \alpha>1$, Theorem 2.1 is due to Kiguradze [19].

If $\mathbb{T}=\mathbb{Z}, r(t)=1, p(t)=0, q(t) \geq 0, f(x)=x^{\alpha}, \alpha>1$, and $\phi(t)=t-1$, then Theorem 2.1 includes Theorem 4.1 in Hooker and Patula [20, Theorem 4.1] and Mingarelli [21].

Suppose that there exists a $C_{\text {rd }}^{1}$ function $\phi$ such that, for $t \in\left[t_{0}, \infty\right)_{\mathbb{T}}$,

$$
\phi(t) r(t)>0, \quad \phi^{\Delta}(t) r(t)=\phi^{\sigma}(t) p(t) .
$$

Then, in this case, we have $P(t) \equiv 0$, and so we do not need to assume the superlinearity conditions (1.3), and so the result applies to linear, sublinear, and superlinear case.

Corollary 2.1 If there exists a $C_{\mathrm{rd}}^{1}$ function $\phi$ such that (2.2) and (2.12) hold, then every solution of equation (1.1) is oscillatory.

If we do not assume superlinearity condition (1.3) and condition (2.12), then we can conclude that all bounded solutions are oscillatory.

Corollary 2.2 If there exists a $C_{\mathrm{rd}}^{1}$ function $\phi$ such that (2.1) and (2.2) hold, then every bounded solution of equation (1.1) is oscillatory.

In the following, we assume that there exists a $C_{\mathrm{rd}}^{1}$ function $\phi$ such that

$$
\int_{t_{0}}^{\infty} \frac{\Delta t}{\phi(t) r(t)}<\infty
$$

holds and establish some sufficient conditions for equation (1.1).

Theorem 2.2 Assume that $f$ satisfies (1.3). If there exists a $C_{\mathrm{rd}}^{1}$ function $\phi$ such that (2.1) and (2.13) hold and if

$$
\int_{t_{0}}^{\infty} \phi^{\sigma}(t) q(t) \Delta t=\infty
$$

and

$$
\int_{t_{0}}^{\infty} \frac{1}{\phi(t) r(t)}\left[\int_{t_{0}}^{t} \phi^{\sigma}(s) q(s) \Delta s\right] \Delta t=\infty
$$

then every solution of equation (1.1) is either oscillatory or tends to zero. 
Proof Assume (1.1) has a nonoscillatory solution $x$ on $\left[t_{0}, \infty\right)_{\mathbb{T}}$. Then, without loss of generality, there is a solution $x$ of $(1.1)$ and a $T \in\left[t_{0}, \infty\right)_{\mathbb{T}}$ such that $x(t)>0$ on $[T, \infty)_{\mathbb{T}}$. As in the proof of Theorem 2.1, by using (2.14), we have that there exists a sufficiently large $T_{1} \geq T$ such that

$$
x^{\Delta}(t)<0, \quad \text { for } t \in\left[T_{1}, \infty\right)_{\mathbb{T}}
$$

and so

$$
w^{\Delta}(t) \leq-\phi^{\sigma}(t) q(t)+\frac{P(t) x^{\Delta}(t)}{f\left(x^{\sigma}(t)\right)} \leq-\phi^{\sigma}(t) q(t),
$$

which yields

$$
\begin{aligned}
\int_{T_{1}}^{t} \phi^{\sigma}(s) q(s) \Delta s & \leq \phi\left(T_{1}\right) \frac{r\left(T_{1}\right) x^{\Delta}\left(T_{1}\right)}{f\left(x\left(T_{1}\right)\right)}-\phi(t) \frac{r(t) x^{\Delta}(t)}{f(x(t))} \\
& \leq-\phi(t) r(t) \frac{x^{\Delta}(t)}{f(x(t))}, \quad \text { for } t \in\left[T_{1}, \infty\right)_{\mathbb{T}} .
\end{aligned}
$$

That is,

$$
\frac{1}{\phi(t) r(t)} \int_{T_{1}}^{t} \phi^{\sigma}(s) q(s) \Delta s \leq-\frac{x^{\Delta}(t)}{f(x(t))}
$$

so integration from $T_{1}$ to $t\left(t \geq T_{1}\right)$ yields

$$
\int_{T_{1}}^{t} \frac{1}{\phi(s) r(s)}\left[\int_{T_{1}}^{s} \phi^{\sigma}(u) q(u) \Delta u\right] \Delta s \leq-\int_{T_{1}}^{t} \frac{x^{\Delta}(s)}{f(x(s))} \Delta s .
$$

Define

$$
F(x(t)):=\int_{x\left(T_{1}\right)}^{x(t)} \frac{d s}{f(s)} .
$$

Then

$$
\begin{aligned}
(F(x(t)))^{\Delta} & =\int_{0}^{1} F^{\prime}\left(x_{h}(t)\right) d h x^{\Delta}(t)=\int_{0}^{1} \frac{1}{f\left(x_{h}(t)\right)} d h x^{\Delta}(t) \\
& \leq \int_{0}^{1} \frac{1}{f(x(t))} d h x^{\Delta}(t)=\frac{x^{\Delta}(t)}{f(x(t))} .
\end{aligned}
$$

Since $x^{\Delta}(t)<0$, we have $\lim _{t \rightarrow \infty} x(t)=L \geq 0$. If we assume $L>0$, then

$$
-F(x(t))=\int_{x(t)}^{x\left(T_{1}\right)} \frac{d s}{f(s)} \leq \int_{L}^{x\left(T_{1}\right)} \frac{d s}{f(s)}<\infty
$$

so

$$
\int_{T_{1}}^{t} \frac{1}{\phi(s) r(s)}\left[\int_{T_{1}}^{s} \phi^{\sigma}(u) q(u) \Delta u\right] \Delta s \leq-\int_{T_{1}}^{t}(F(x(s)))^{\Delta} \Delta s=-F(x(t))<\infty,
$$

and let $t \rightarrow \infty$ to get a contradiction to (2.15). This completes the proof. 
Corollary 2.3 If there exists a $C_{\mathrm{rd}}^{1}$ function $\phi$ such that (2.12), (2.13), (2.14), and (2.15) hold, then every solution of equation (1.1) is either oscillatory or tends to zero.

Corollary 2.4 If there exists a $C_{\mathrm{rd}}^{1}$ function $\phi$ such that (2.1), (2.13), (2.14), and (2.15) hold, then every bounded solution of equation (1.1) is either oscillatory or tends to zero.

Next we present oscillation criteria for equation (1.1) where $f$ satisfies sublinearity condition (1.4).

Theorem 2.3 Assume that $f$ satisfies (1.4). If there exists a $C_{\mathrm{rd}}^{1}$ function $\phi$ such that (2.12), (2.13), (2.14), and (2.15), then every solution of equation (1.1) is oscillatory.

Proof Assume (1.1) has a nonoscillatory solution $x$ on $\left[t_{0}, \infty\right)_{\mathbb{T}}$. Then, without loss of generality, there is a solution $x$ of $(1.1)$ and a $T \in\left[t_{0}, \infty\right)_{\mathbb{T}}$ such that $x(t)>0$ on $[T, \infty)_{\mathbb{T}}$. As in the proof of Theorems 2.1 and 2.2, we have that there exists a sufficiently large $T_{1} \geq T$ such that

$$
x^{\Delta}(t)<0, \quad \text { for } t \in\left[T_{1}, \infty\right)_{\mathbb{T}},
$$

and, from (2.16) and (2.17), we get

$$
\int_{T_{1}}^{t} \frac{1}{\phi(s) r(s)}\left[\int_{T_{1}}^{s} \phi^{\sigma}(u) q(u) \Delta u\right] \Delta s \leq-\int_{T_{1}}^{t}(F(x(s)))^{\Delta} \Delta s=-F(x(t)) .
$$

Since $x^{\Delta}(t)<0$, then $\lim _{t \rightarrow \infty} x(t) \geq 0$ and so

$$
-F(x(t))=\int_{x(t)}^{x\left(T_{1}\right)} \frac{d s}{f(s)} \leq \int_{0}^{x\left(T_{1}\right)} \frac{d s}{f(s)} \stackrel{(1.4)}{<} \infty .
$$

Then

$$
\int_{T_{1}}^{t} \frac{1}{\phi(s) r(s)}\left[\int_{T_{1}}^{s} \phi^{\sigma}(u) q(u) \Delta u\right] \Delta s<\infty
$$

which is a contradiction to (2.15). This completes the proof.

Theorem 2.4 Assume that $f$ satisfies (1.4). If there exists a $C_{\mathrm{rd}}^{1}$ function $\phi$ such that (2.1), (2.13), (2.14), and (2.15) hold, then every bounded solution of equation (1.1) is oscillatory.

The results are very general. With appropriate choices of $\phi(t)$, we can obtain several sufficient conditions for the oscillation of equation (1.1).

\section{Examples}

In this section, we give two examples to illustrate our main results. We note that in the first example, all of the coefficient functions $r(t), p(t), q(t)$ change sign for arbitrarily large values of $t$, and the function $f$ may be linear, superlinear, or sublinear, since we do not assume nonlinearity conditions (1.3) and (1.4). 
Example 3.1 Let $t_{0}>0$ and $\mathbb{T}$ be a discrete time scale satisfying $\mu>0$ on $\mathbb{T}$. Consider the second-order nonlinear dynamic equation with damping

$$
\left(r\left(t_{n}\right) x^{\Delta}\left(t_{n}\right)\right)^{\Delta}+p\left(t_{n}\right) x^{\Delta}\left(t_{n}\right)+q\left(t_{n}\right) f\left(x^{\sigma}\left(t_{n}\right)\right)=0,
$$

where $f$ satisfies $f^{\prime}(x)>0$ and $x f(x)>0$ for $x \neq 0$. Define, for $t_{n} \in \mathbb{T}, n \in \mathbb{N}$,

$$
\phi\left(t_{n}\right):=(-1)^{n} t_{n}^{\beta_{1}}, \quad r\left(t_{n}\right):=\frac{(-1)^{n}}{t_{n}^{\beta_{2}}}, \quad p\left(t_{n}\right):=\frac{(-1)^{n}}{t_{n}^{\beta_{2}} \mu\left(t_{n}\right)}\left(1+\left(\frac{t_{n}}{t_{n+1}}\right)^{\beta_{1}}\right)
$$

and

$$
q\left(t_{n}\right):=\frac{1}{t_{n+1}^{\beta_{1}}}\left(\frac{1}{t_{n} t_{n+1}}-\frac{(-1)^{n}}{t_{n}^{\beta_{3}}}\right)
$$

where $\beta_{i} \in \mathbb{R}, i=1,2,3$ such that $\beta_{1}-\beta_{2} \leq 1$ and $\beta_{3} \leq 1$. Therefore,

$$
\phi\left(t_{n}\right) r\left(t_{n}\right)=t_{n}^{\beta_{1}-\beta_{2}}>0, \quad \phi^{\Delta}\left(t_{n}\right) r\left(t_{n}\right)=\phi^{\sigma}\left(t_{n}\right) p\left(t_{n}\right)
$$

so (2.12) is satisfied. Also,

$$
\int_{t_{0}}^{\infty} \frac{\Delta t_{n}}{\phi\left(t_{n}\right) r\left(t_{n}\right)}=\int_{t_{0}}^{\infty} \frac{\Delta t_{n}}{t_{n}^{\beta_{1}-\beta_{2}}}=\infty
$$

and

$$
\int_{t_{0}}^{\infty} \phi^{\sigma}\left(t_{n}\right) q\left(t_{n}\right) \Delta t_{n}=\int_{t_{0}}^{\infty}\left(\frac{1}{t_{n}^{\beta_{3}}}-\frac{(-1)^{n}}{t_{n} t_{n+1}}\right) \Delta t_{n}=\infty
$$

by Example 5.60 in [3]. Then, by Theorem 2.1, every solution of equation (3.1) is oscillatory.

Example 3.2 Let $\mathbb{T}=\mathbb{N}$ and consider the difference equation

$$
\Delta(r(n) \Delta x(n))+p(n) \Delta x(n)+q(n) f(x(n+1))=0, \quad n \geq n_{0}>1
$$

where $f$ satisfies $f^{\prime}(x)>0$ and $x f(x)>0$ for $x \neq 0$ and nonlinearity condition (1.3). Define

$$
\phi(n):=(n-1)^{\beta}, \quad r(n):=n^{\delta-\beta}, \quad p(n):=0
$$

and

$$
q(n):=\frac{a}{n^{\alpha+\gamma}}+\frac{b(-1)^{n}}{n^{\gamma}}
$$

where $\alpha, \delta \in[0,1]$ and $a, b, \gamma, \beta \in \mathbb{R}$. It is easy to see that (2.1) and $\int_{t_{0}}^{\infty} \frac{\Delta t}{\phi(t) r(t)}=\infty$ hold. Note that

$$
\int_{t_{0}}^{\infty} \sigma^{\beta}(t) q(t) \Delta t=\sum_{k=n_{0}}^{\infty}\left(\frac{a}{k^{\alpha+\gamma-\beta}}+\frac{b(-1)^{k}}{k^{\gamma-\beta}}\right) .
$$


If $\beta=\gamma$, we get

$$
\int_{t_{0}}^{\infty} \sigma^{\beta}(t) q(t) \Delta t=\infty .
$$

Then, by Theorem 2.1, every solution of equation (3.2) is oscillatory.

\section{Competing interests}

The author declares that he has no competing interests.

Received: 13 June 2012 Accepted: 13 September 2012 Published: 2 October 2012

\section{References}

1. Hilger, S: Analysis on measure chains - a unified approach to continuous and discrete calculus. Results Math. 18, 18-56 (1990)

2. Bohner, M, Peterson, A: Dynamic Equation on Time Scales: An Introduction with Applications. Birkhäuser, Boston (2001)

3. Bohner, M, Peterson, A (eds.): Advances in Dynamic Equations on Time Scales. Birkhäuser, Boston (2003)

4. Akin-Bohner, E, Bohner, M, Saker, SH: Oscillation criteria for a certain class of second order Emden-Fowler dynamic equations. Electron. Trans. Numer. Anal. 27, 1-12 (2007)

5. Bohner, M, Hassan, TS: Oscillation and boundedness of solutions to first and second order forced functional dynamic equations with mixed nonlinearities. Appl. Anal. Discrete Math. 3, 242-252 (2009)

6. Erbe, L, Hassan, TS, Peterson, A: Oscillation criteria for nonlinear damped dynamic equations on time scales. Appl. Math. Comput. 203, 343-357 (2008)

7. Erbe, L, Hassan, TS, Peterson, A: Oscillation criteria for nonlinear functional neutral dynamic equations on time scales. J. Differ. Equ. Appl. 15, 1097-1116 (2009)

8. Erbe, L, Hassan, TS, Peterson, A, Saker, SH: Oscillation criteria for half-linear delay dynamic equations on time scales. Nonlinear Dyn. Syst. Theory 9(1), 51-68 (2009)

9. Erbe, L, Hassan, TS, Peterson, A, Saker, SH: Oscillation criteria for sublinear half-linear delay dynamic equations on time scales. Int. J. Differ. Equ. 3, 227-245 (2008)

10. Hassan, TS: Oscillation criteria for half-linear dynamic equations on time scales. J. Math. Anal. Appl. 345, 176-185 (2008)

11. Saker, SH: Oscillation of nonlinear dynamic equations on time scales. Appl. Math. Comput. 148, 81-91 (2004)

12. Erbe, L: Oscillation criteria for second order linear equations on a time scale. Can. Appl. Math. Q. 9, 346-375 (2001)

13. Erbe, $L$, Peterson, $A$ : Boundedness and oscillation for nonlinear dynamic equation on a time scale. Proc. Am. Math. Soc. $132,735-744$ (2003)

14. Erbe, L, Peterson, A: An oscillation result for a nonlinear dynamic equation on a time scale. Can. Appl. Math. Q. 11, 143-159 (2003)

15. Bohner, $M$, Erbe, L, Peterson, A: Oscillation for nonlinear second order dynamic equations on a time scale. J. Math. Anal. Appl. 301, 491-507 (2005)

16. Baoguo, J, Erbe, L, Peterson, A: Kiguradze-type oscillation theorems for second order superlinear dynamic equations on time scales. Can. Math. Bull. 54, 580-592 (2011)

17. Hassan, TS, Erbe, L, Peterson, A: Oscillation of second order superlinear dynamic equations with damping on time scales. Comput. Math. Appl. 59, 550-558 (2010)

18. Hassan, TS, Erbe, L, Peterson, A: Oscillation criteria for second order sublinear dynamic equations with damping term. J. Differ. Equ. Appl. 17, 505-523 (2011)

19. Kiguradze, IT: A note on the oscillation of solutions of the equation $u^{\prime \prime}+a(t) u^{n} \operatorname{sgn} u=0$. Čas. Pěst. Mat. $92,343-350$ (1967)

20. Hooker, JW, Patula, WT: A second order nonlinear difference equation: oscillation and asymptotic behavior. J. Math. Anal. Appl. 91, 9-29 (1983)

21. Mingarelli, AB: Volterra-Stieltjes Integral Equations and Generalized Differential Equations. Lecture Notes in Mathematics, vol. 989. Springer, Berlin (1983)

22. Atkinson, FV: On second order nonlinear oscillations. Pac. J. Math. 5, 643-647 (1955)

23. Wintner, A: A criterion of oscillatory stability. Q. Appl. Math. 7, 115-117 (1949)

24. Bartle, RG: The Elements of Real Analysis, 2nd edn. Wiley, New York (1976)

25. Belohorec, S: Oscillatory solutions of certain nonlinear differential equations of second order. Mat.-Fyz. Čas. Slovensk. Akad. Vied 11, 250-255 (1961)

26. Belohorec, S: Two remarks on the properties of solutions of a nonlinear differential equation. Acta Fac. Rerum Nat. Univ. Comen. Math. 22, 19-26 (1969)

27. Fite, WB: Concerning the zeros of solutions of certain differential equations. Trans. Am. Math. Soc. 19, 341-352 (1917)

28. Erbe, L, Baoguo, J, Peterson, A: Belohorec-type oscillation theorem for second order sublinear dynamic equations on time scales. Math. Nachr. 284, 1658-1668 (2011)

29. Wong, JS: Oscillation criteria for second order nonlinear differential equations involving general means. J. Math. Anal. Appl. 247, 489-505 (2000)

30. Keller, S: Asymptotisches Verhalten Invarianter Faserbü ndel bei Diskretisierung und Mittelwertbildung im Rahmen der Analysis auf Zeitskalen. PhD thesis, Universität Augsburg (1999)

31. Leighton, W: On self-adjoint differential equations of second order. J. Lond. Math. Soc. 27, 37-47 (1952) 
32. Hassan, TS, Erbe, L, Peterson, A: Forced oscillation of second order functional differential equations with mixed nonlinearities. Acta Math. Sci., Ser. B 31(2), 613-626 (2011)

33. Hassan, TS: Interval oscillation for second order nonlinear differential equations with a damping term. Serdica Math. J. $34,715-732(2008)$

doi:10.1186/1687-1847-2012-171

Cite this article as: Hassan: Oscillation criteria for second-order nonlinear dynamic equations. Advances in Difference Equations 2012 2012:171

Submit your manuscript to a SpringerOpen ${ }^{\circ}$ journal and benefit from:

- Convenient online submission

- Rigorous peer review

- Immediate publication on acceptance

- Open access: articles freely available online

- High visibility within the field

- Retaining the copyright to your article

Submit your next manuscript at $>$ springeropen.com 